\title{
Latitudinal and longitudinal displacement of cusp ion precipitation controlled by IMF $B_{y}$ and $B_{z}$
}

\author{
Keiko T. Asai ${ }^{1}$, Kiyoshi Maezawa ${ }^{2}$, Toshifumi Mukai ${ }^{2}$, and Hajime Hayakawa ${ }^{2}$ \\ ${ }^{1}$ National Institute of Information and Communications Technology, 4-2-1 Nukuikita, Koganei, Tokyo 184-8795, Japan \\ ${ }^{2}$ Institute of Space and Astronautical Science, 3-1-1 Yoshinodai, Sagamihara, Kanagawa 229-8510, Japan
}

(Received December 28, 2004; Revised April 25, 2005; Accepted April 25, 2005)

\begin{abstract}
Dependence of the location of the cusp precipitation on the orientation of interplanetary magnetic field (IMF) is investigated using data from the Akebono satellite taken at altitudes of several thousands of $\mathrm{km}$. More than a hundred cusp precipitation events have been identified with the low-energy particle detector (LEP) onboard Akebono. The observed energy spectra of ions precipitating at the cusp are dispersed by the convection motion of field lines. We pay special attention to the location where precipitating ions have the highest energy in the energy dispersion curve as recorded along the satellite's path; such location would represent the one nearest to the foot point of the dayside reconnection line (we here assume that the cusp ion injection is triggered by the magnetopause reconnection). We study this location as a proxy for the foot point of the reconnection line and call it "ion entry point" in this paper. Our analysis shows that the location of the "ion entry point" has a strong dependence on the sign and magnitude of IMF $B_{y}$ and $B_{z} . B_{y}$ and $B_{z}$ have almost orthogonal effects on the location of the entry point, with the latitudinal displacement linearly related to $B_{z}$, and the longitudinal displacement linearly related to $B_{y}$. We find that the $B_{z}$ dependence of the ion entry point is represented by a single regression line for entire range of $B_{z}$ irrespective of its north/south polarity. We also find that the $B_{y}$ dependence is larger for positive $B_{z}$ than for negative $B_{z}$. The distribution of ion entry points for positive IMF $B_{y}$ is almost a mirror image of that for negative IMF $B_{y}$ with respect to the noon-midnight meridian plane. We discuss the dependence of the "ion entry point" on the IMF from the viewpoint of where and how the magnetopause reconnection (merging) occurs for various orientations of IMF.
\end{abstract}

Key words: Cusp, cusp precipitation, magnetic reconnection, dayside merging, interplanetary magnetic field.

\section{Introduction}

The polar cusp is one of the most interesting regions of the Earth's magnetosphere. It is defined as a region of weak magnetic field around a theoretical neutral point formed in each hemisphere when the Earth's dipole magnetic field is compressed by the solar wind. Actually, the location of the polar cusp is not uniquely determined by the solar wind flow but it also depends on the IMF conditions and on magnetospheric convection. Magnetospheric convection is driven by dayside reconnection and its low-altitude signature starts from the polar cusp, since the dayside reconnection that occurs on the dayside magnetopause is connected to the polar cusp by reconnected field lines. Through the polar cusp, magnetosheath plasma is injected along the reconnected field lines, and precipitates into the ionosphere. Injected plasma affects various phenomena occurring at the ionospheric level; for example, it affects ionospheric conductivity, cusp auroral emission, field aligned currents, ionospheric plasma heating, etc.

Polar orbiting satellites such as Akebono provide an important tool to survey the region below the polar cusp, called the low-altitude cusp, in order to obtain information on

Copy right(C) The Society of Geomagnetism and Earth, Planetary and Space Sciences (SGEPSS); The Seismological Society of Japan; The Volcanological Society of Japan; The Geodetic Society of Japan; The Japanese Society for Planetary Sciences; TERRAPUB the dayside reconnection. This is because terrestrial magnetic field lines on the dayside magnetopause all converge into the polar cusp and connect to the low-latitude cusp, so that the information about the processes occurring on the dayside magnetopause also converges into the low-altitude cusp.

The low-altitude cusp is known to be located around 12 LT but the study of its location from satellite observations has some ambiguity because of the finite extent of the cusp. Furthermore, its location requires careful statistics since it is known to vary with IMF orientations. On average, low-altitude satellite and ground-based observations indicate that the low-altitude cusp has a latitudinal width of 1-2 degrees and extends in longitude from about 10 to 14 MLT (magnetic local time) (Newell et al., 1989; Aparicio et al., 1991; Kremser and Rundin, 1990; Karlson et al., 1996). There has been indication that the latitudinal extent narrows during southward IMF and widens during northward IMF (Newell and Meng, 1987).

Recent in situ observations of the polar cusp by Polar (Chandler et al., 1999; Stubbs et al., 2004; Faruggia et al., 2004) and Cluster (Twitty et al., 2004) spacecraft have added new evidence for the IMF control of the cusp location at high altitudes. Crooker et al. (1987) have reported that the location of the cusp changes in the azimuthal direction with IMF $B_{y}$, based on the magnetic field observations of 
the dayside magnetopause. However, these observations do not cover the whole dayside magnetopause, since the dayside magnetopause is too wide to be completely surveyed by spacecraft.

On the other hand, the low-altitude observations of the polar cusp have the advantage that all the information about the dayside magnetopause converges into the low-altitude cusp. However, they also have some disadvantages. Firstly, the observation is influenced by ionospheric/magnetospheric convection. The convective motion of field lines disperses the location of precipitating ions as they drift in the direction of convection, and the degree of dispersion depends on ion energy. This energy dispersion structure was reported earlier by Rosenbauer et al. (1975) and Reiff et al. (1977). This means that the identification of the cusp depends on the ion energy that the particle detector onboard the satellite looks at. At higher energies, the location of the cusp is nearer to the foot of the injection point (i.e. the foot of the field line connected to the reconnection line), while at lower energies, the location of the cusp should appear downstream of the injection point in terms of convection. The direction of ionospheric convection is known to be controlled by the IMF orientation. In particular, the ionospheric convection around noon is directed poleward during southward IMF and equatorward during northward IMF (cf., e.g., Maezawa, 1976). Direction of ionospheric convection as a function of IMF $B_{y}$ and $B_{z}$ has been summarized by Heelis (1984), Cowley et al. (1991), Lockwood et al. (1995), Ridley et al. (1998), Maynard et al. (2001), Fuselier et al. (2002), Sandholt and Farrugia (2003), among others.

Another disadvantage of low altitude observations is the difficulty in distinguishing cusp from other types of ion precipitation. In this regard, Newell and Meng (1988), Newell et al. (1991a, 1991b) and Lockwood et al. (1997) classified dayside precipitation for southward IMF conditions in detail, based on observations made by the DMSP satellites. Onsager et al. $(1993,1995)$ theoretically modeled ion injections triggered by dayside reconnection for the southward IMF and reported that the energy-dispersed structures in modeled ion precipitation are consistent with the DMSP satellite observations of cusp ions. Cowley (1982) and Lockwood et al. (1996) theoretically modeled the magnetic slow shock caused by dayside reconnection and noted that the dayside ion precipitation observed at low altitudes includes a small amount of magnetospheric ions accelerated through the slow shock that mix with the main component of magnetosheath origin.

In the present study, we minimize ambiguities in determining cusp location from the Akebono observations at middle-altitudes of several thousands km. Akebono cusp observations are not confined to the region around noon but are distributed also in the regions far from the noon, both on the dawnside and on the duskside. Such a wide distribution motivated us to do the present study and to be sensitive about the determination of the location of the cusp precipitation.

Asai et al. (1999), hereafter called Paper 1, investigated the dependence of cusp-ion latitudinal displacements on IMF $B_{z}$. In Paper 1, the high-energy boundary of ion dis- persion, called the starting point of cusp precipitation, represented the point closest to the separatrix of reconnected magnetic filed lines. In spite of the simple definition of this point, a clear latitudinal displacement controlled by IMF $B_{z}$ was obtained for a wide range of the $B_{z}$ component, including the cases of northward IMF orientations. It was found that thus defined starting latitude of cusp precipitation is correlated almost linearly with IMF $B_{z}$ regardless of its sign, and that the solar wind velocity $\left(V_{s w}\right)$ weakly affects its latitude.

In the present paper, we elaborate on the IMF dependence of the cusp location including the influence of IMF $B_{y}$. IMF $B_{y}$ and $B_{z}$ are important for the dayside reconnection because these components are tangent to the surface of the dayside magnetopause. We discuss the dislocation of the cusp and the changes in the convection direction owing to the magnetopause reconnection from our results.

\section{Data Analysis}

In this paper we define the "ion entry point" for cusp ion precipitation from the plasma data collected by the Japanese polar-orbiting satellite, Akebono. Akebono was launched on February 22, 1989, into the semi-polar orbit with the apogee of $10,500 \mathrm{~km}$, the perigee of $270 \mathrm{~km}$, and the inclination of $75^{\circ}$. The present apogee altitude is lower than $8,000 \mathrm{~km}$ while the perigee altitude remains the same as the initial one.

\subsection{Database}

Our data set consists of measurements taken in the years 1989-1993 with the low energy charged particle detectors (LEP) and the electric field detectors (EFD) onboard Akebono. The time resolution of these data is $8 \mathrm{sec}$ (one spin period). The energy coverage of the LEP is from $10 \mathrm{eV}$ to 16 $\mathrm{keV}$ for electrons and from $13 \mathrm{eV} / Q$ to $20 \mathrm{keV} / Q$ for ions. The LEP data are limited in the latitudinal coverage, i.e., they are taken only poleward of $\pm 60^{\circ}$ invariant latitude. In this paper, the position of the satellite (its latitude and magnetic local time) is represented in corrected geomagnetic coordinates (Hakura, 1965). Calibrated LEP data are available in the form of differential energy flux $\left[\mathrm{eV} / \mathrm{cm}^{2} / \mathrm{sr} / \mathrm{sec} / \Delta \mathrm{eV}\right]$ for 29 energy channels divided logarithmically and for 18 pitch-angle channels in the range of $0-180^{\circ}$. The EFD measures 2-dimensional electric field with two sets of double probes in the GSM $Y-Z$ plain, which is perpendicular to the spin axis of the satellite. The calibrated EFD data are available in the form of the Cartesian three components in GSM coordinates. The GSM- $X$ component is derived under the assumption that there exists no electric field component parallel to the ambient magnetic field. $\boldsymbol{E} \times \boldsymbol{B}$ convection velocities at the altitude of $120 \mathrm{~km}$ are calculated from the electric field data using the IGRF 1985 model for the field line projection. The details of the LEP and EFD instruments are given in Mukai et al. (1990) and Hayakawa et al. (1990), respectively. For IMF and solar wind parameters, we use hourly values from IMP 8 distributed by NSSDC.

\subsection{Definition of "ion entry point"}

Cusp ion precipitation detected by a low- or middlealtitude satellite shows distinguishing features such as energy-dispersion structures caused by magnetospheric (ionospheric) convection. This kind of energy dispersion 
structures can be observed by a satellite whose orbit is at an oblique angle to the convection direction. Such a dispersion structure often appears as latitudinal dispersion in which the energy of ions decreases poleward under poleward ionospheric convection. Poleward convection at noon is a part of the two-cell convection pattern typically appearing during southward IMF. Rosenbauer et al. (1975) have reported this energy dispersion (they called this type of precipitation "mantle") and Reiff et al. (1977) calculated the distance of the ion source from the energy dispersion curve from satellite observations made at altitudes of about 1,300 $\mathrm{km}$. Woch and Lundin (1992) have reported energy dispersions observed by the Viking spacecraft.

Figure 1 is a schematic diagram illustrating the "ion entry point" that we define in this paper from the data of cusp ion precipitation. Injected ions from the dayside polar cusp are dispersed by magnetospheric convection. Convection occurs perpendicular to the field lines, and the convection velocity, $\boldsymbol{V}_{\mathbf{c}}=\boldsymbol{E}_{\mathbf{c}} \times \boldsymbol{B} / B^{2}$, is common to all the precipitating ions (and electrons). However, at the ionospheric altitudes, ions with higher energies are found close to the foot of the injection point because of their shorter travel time along the field line, while ions with lower energies are observed farther from the injection point because of their longer travel time, during which ions are convected perpendicular to the field line. Such an energy dispersion structure of injected ions should always be seen except when the convection velocity vanishes or when it is exactly perpendicular to the satellite path. The "ion entry point" is defined as the location where highest energy ions are observed along the satellite path. We consider the magnetic field lines threading the "ion entry point" to represent the field lines nearest to the foot of the reconnection line, where the ions are injected into the magnetosphere). In other words, the ion entry point marks the most recently reconnected field lines encountered along the satellite path.

We show four representative cases of the cusp ion energy dispersion for four different IMF conditions in Figs. 25. Cases 1 and 2 occurred in the southern hemisphere
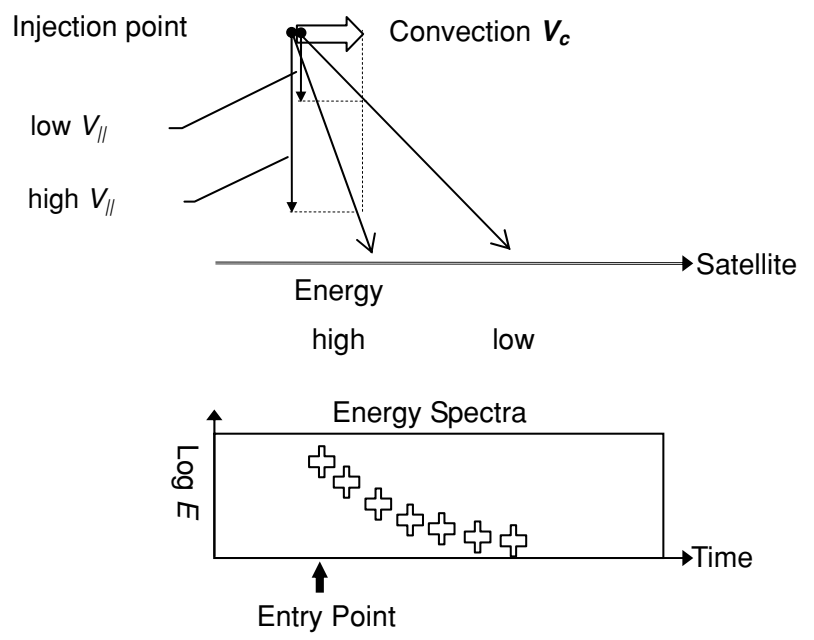

Fig. 1. Definition of "ion entry point" from observation of cusp ion precipitation. The ion entry point is the location where highest energy ions precipitate in the energy dispersion structure of ion precipitation. for southward and northward IMF conditions, respectively. Cases 3 and 4 occurred in the northern hemisphere for positive and negative IMF $B_{y}$ conditions, respectively. The ion entry points can be clearly identified for each of four cases.

The data in Fig. 2 show the case of southward IMF on January 31, 1991 (Case 1). The top two color diagrams give the differential energy flux $\left[\mathrm{eV} / \mathrm{cm}^{2} / \mathrm{sr} / \mathrm{sec} / \Delta \mathrm{eV}\right]$ of electrons (upper panel) and ions (lower panel). Shown is the maximum differential flux among six pitch angle bins from $0^{\circ}$ to $180^{\circ}$. The middle three panels show the integrated flux, average energy, and density of downward precipitating electrons (blue) and ions (green), corresponding to pitch angles of 90-180 in the southern hemisphere. The bottom panels show the three electric-field components obtained by the double probes. The blue vectors overlaid on the $E_{y}$ plot indicate $\boldsymbol{E} \times \boldsymbol{B}$ convection velocities at an altitude of 120 $\mathrm{km}$ projected along the field lines; $V_{x}$ is positive upward (sunward) and $V_{y}$ is positive toward left (duskward). The same vectors are plotted along the Akebono orbit projected onto the ionosphere at the bottom of the figure. Akebono traveled poleward near geomagnetic noon in the southern polar region. It encountered the cusp precipitation at latitudes of $72-76^{\circ}$ in the corrected geomagnetic coordinates at 0738-0745 UT. The ion energy was highest at the latitude of about $72^{\circ}$ and decreased with increasing latitude. This flux peak occurred a small distance away from the location of the energy peak. In addition, we note that the plasma convection obtained from the electric field was directed poleward for the corresponding time interval, and the satellite trajectory was almost parallel to the convection direction. The hourly values of solar wind parameters were: ion temperature $45,300 \mathrm{~K}$, density 20.4 /cc, velocity 373 $\mathrm{km} / \mathrm{s}$, and IMF vector components $B_{x}, B_{y}$, and $B_{z},-5.9$, -3.7 , and $-8.0 \mathrm{nT}$, respectively. It is known that during periods of such a strong southward IMF, a two-cell type convection pattern usually occurs in the polar region, which is consistent with the observed poleward convection near noon. The existence of ion precipitation having a latitudinal energy dispersion within the region of poleward convection is a typical signature for a strong southward IMF.

Figure 3 shows the case of northward IMF on October 5 , 1989 (Case 2). A cusp precipitation event was encountered at 0826-0831 UT. The satellite trajectory was similar to that in Case 1 but the cusp precipitation was located at latitudes of $79-83^{\circ}$, significantly higher than that in Case 1. The highest energy ion precipitation occurred at the latitude of about $83^{\circ}$, with energies decreasing equatorward. Since this sense of energy dispersion is opposite to that in Case, 1 , it is expected that the convection direction is also opposite to that in Case 1 , which is indeed the case as seen from the sunward direction of $\boldsymbol{E} \times \boldsymbol{B}$ convection vectors. Sunward convection around noon is consistent with the 4-cell convection pattern in the polar region, which is known to appear during the period of strong northward IMF. The solar wind hourly values for this period were: ion temperature 22,000 K, density 6.9 /cc, velocity $367 \mathrm{~km} / \mathrm{s}$, and IMF vector $(3.5,-3.0,4.3) \mathrm{nT}$. The existence of ion precipitation having a reverse latitudinal energy dispersion within the region of equatorward convection is a typical signature for a strong northward IMF. 

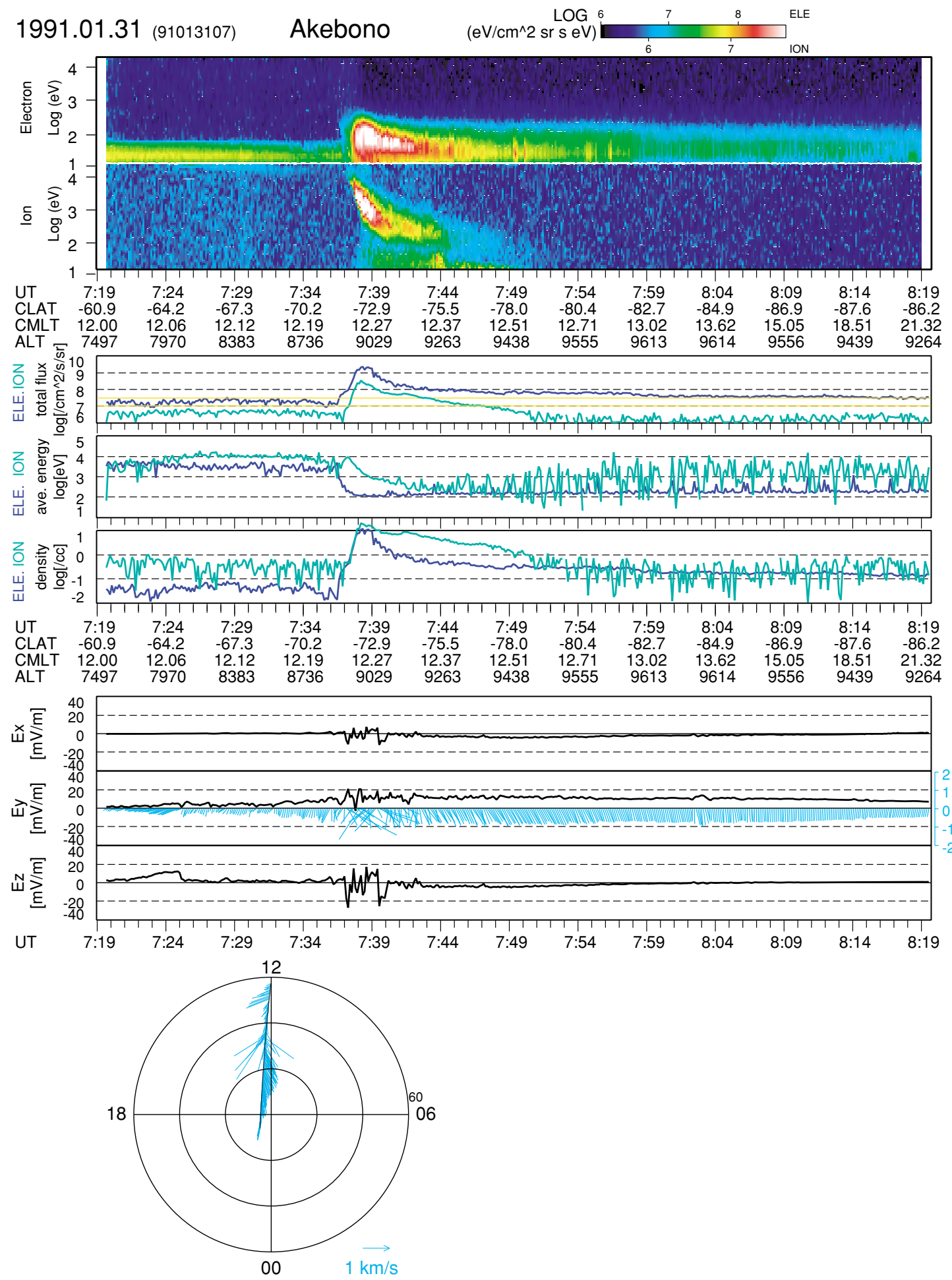

SW $4.5 \mathrm{E}+04 \mathrm{~K} 20.4 / \mathrm{cc} 373.0 \mathrm{~km} / \mathrm{s} \quad \operatorname{IMF}(-5.9-3.7-8.0) \mathrm{nT} \quad \mathrm{Kp}=3+\quad[111]$

Fig. 2. An example of a cusp ion precipitation event observed in the southern hemisphere for the case of southward IMF on January 31, 1991 (Case 1). The Akebono trajectory is directed poleward near noon. A typical cusp ion precipitation with a clear energy dispersion is recorded, with the highest energy ions precipitating at latitude 72 and energies decreasing poleward. The automatically determined cusp precipitation interval for this case is 0737:56-0744:44 UT.

Figures 4 and 5 show, respectively, the data obtained during the period of positive IMF $B_{y}$ on July 25, 1991 (Case 3 ) and during the period of negative IMF $B_{y}$ on August 6 , 1991 (Case 4). In both cases, Akebono traveled in the dayside northern polar region from dawnside toward duskside.
Hourly solar wind values for Case 3 were: ion temperature $21,100 \mathrm{~K}$, density $7.8 / \mathrm{cc}$, velocity $444 \mathrm{~km} / \mathrm{s}$, and IMF vector $(2.6,-7.2,1.4) \mathrm{nT}$. Hourly solar wind values for Case 4 were: ion temperature $86,700 \mathrm{~K}$, density 10.2 /cc, velocity $490 \mathrm{~km} / \mathrm{s}$, and IMF vector $(-1.6,7.7,3.7) \mathrm{nT}$. Note 


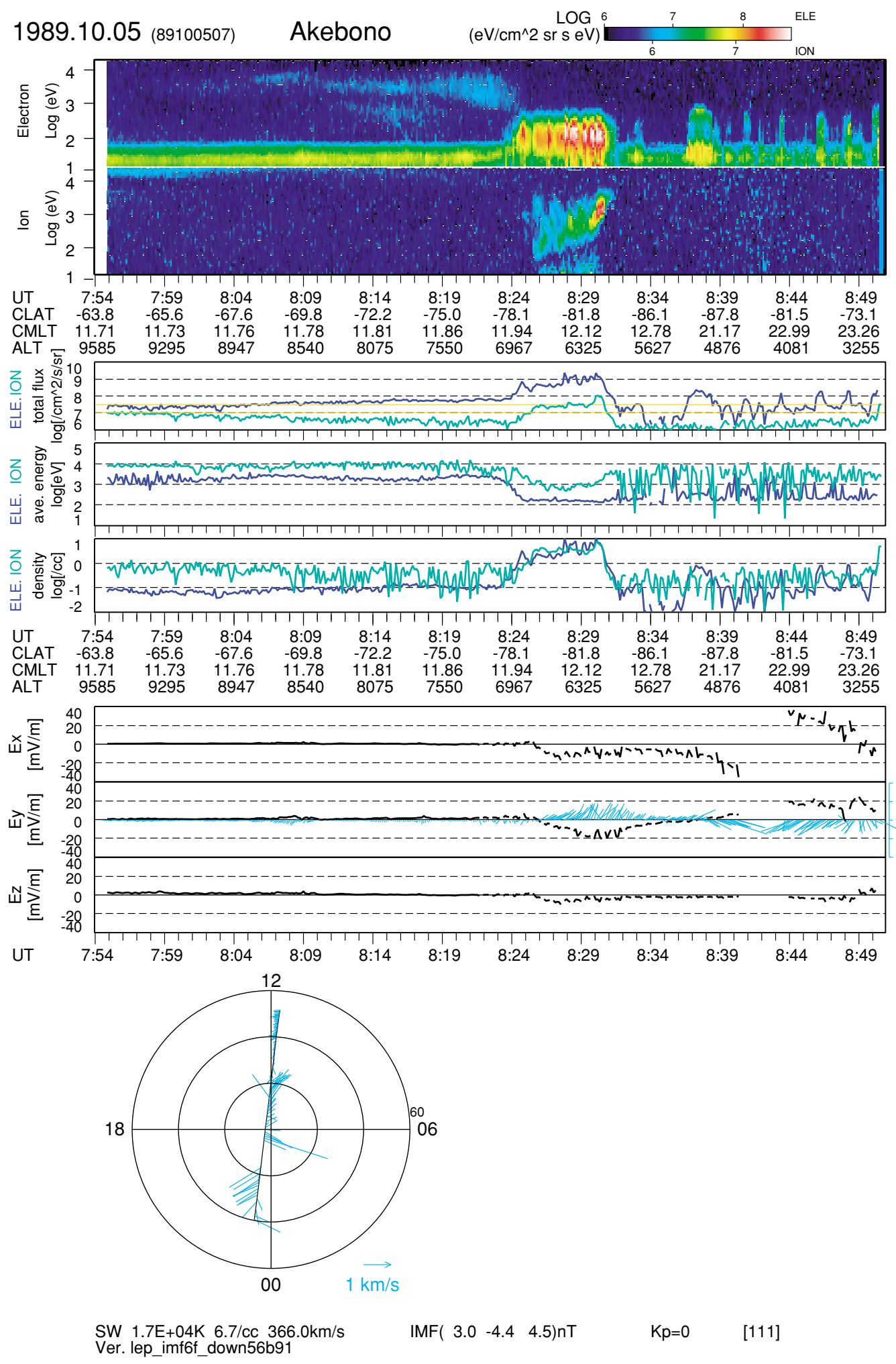

Fig. 3. The same as Fig. 2, but for the northward IMF case on October 5, 1989, observed in the southern hemisphere (Case 2). The satellite trajectory is almost the same as Case 1, but the data show cusp ion precipitation with clear energy dispersion with energies decreasing equatorward. The automatically determined cusp interval is 0825:40-0830:44 UT.

that there is a large difference in the IMF $B_{y}$ component between these two cases. Cusp ion precipitation in Case 3 was observed in the latitudinal range of $78-80^{\circ}$ and in the local time range of 10.4-12.2 MLT (2252-2259 UT). Cusp ion precipitation in Case 4 was observed in the latitudinal range of $76-78^{\circ}$ and in the local time range of 12.9-13.9 MLT
(2242-2251 UT). In both cases, ion precipitation shows a clear energy dispersion structure along the satellite path which is nearly aligned with the east-west direction. However, the sense of energy dispersion is opposite and looks like mirror images of each other. $\boldsymbol{E} \times \boldsymbol{B}$ convection estimated from the electric field data agrees with the direction 

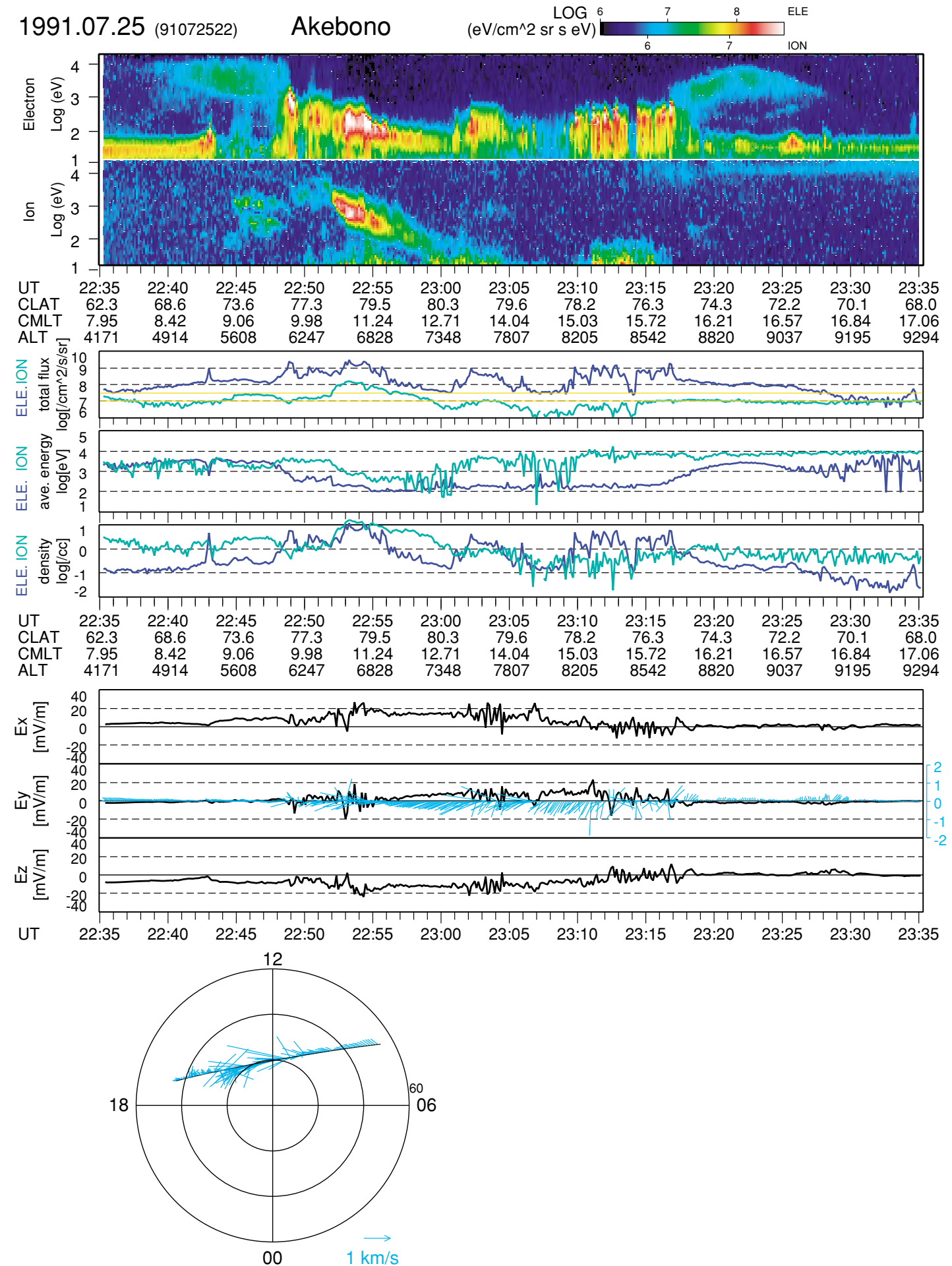

SW 2.1E+04K 7.8/cc $444.0 \mathrm{~km} / \mathrm{s}$ Ver. lep_imf6f_down56b91

Fig. 4. The same as Fig. 2, but observed in the northern hemisphere for the case of dawnward IMF on July 25, 1991 (Case 3). The satellite trajectory is directed duskward on the dayside. The data show cusp ion precipitation with a clear energy dispersion with energies decreasing duskward. The automatically determined cusp interval is 2251:56-2258:20 UT.

of convection suggested by the ion energy dispersion structure in each case. The occurrence of ion precipitation having an energy dispersion within the region of east-west convection is a typical signature for strong IMF $B_{y}$ periods. The mainly east-west direction of convection around noon is consistent with the three-cell convection pattern observed for the periods of large IMF $B_{y}$.

Cases 1 to 4 show that the ion energy dispersion structure can be understood as a spatial structure controlled by IMF. The direction of ion convection around noon strongly depends on the sign of IMF $B_{y}$ and $B_{z}$ components. Cusp precipitation is dispersed over a few degrees of latitude or over 

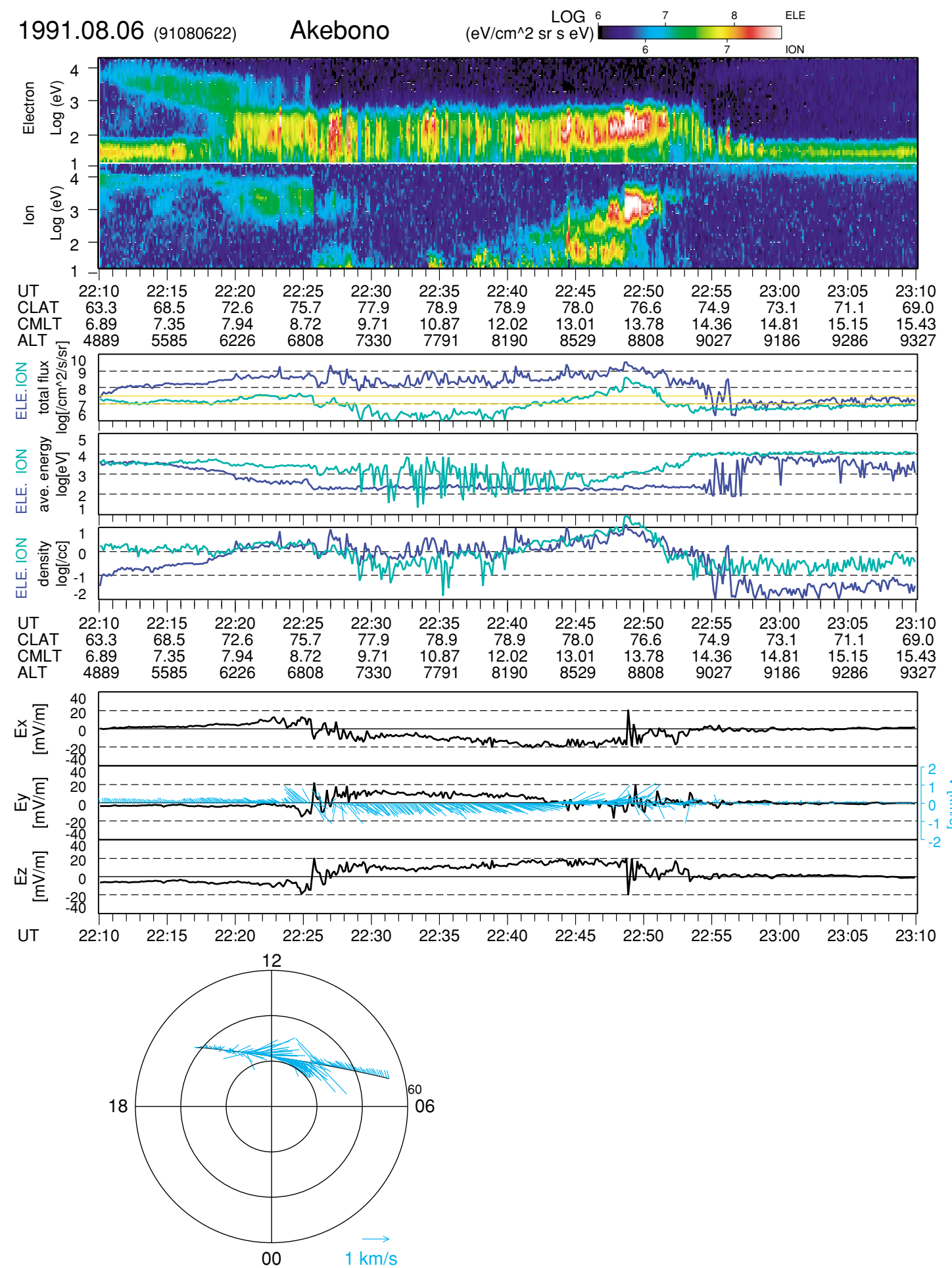

SW $8.7 \mathrm{E}+04 \mathrm{~K} \quad 10.2 / \mathrm{cc} 490.0 \mathrm{~km} / \mathrm{s}$ Ver. lep_imf6f down56b91

Fig. 5. The same as Fig. 4, but for the case of duskward IMF on August 6, 1991 (Case 4). The satellite trajectory is directed duskward on the dayside. The data show cusp ion precipitation with a clear energy dispersion with energies decreasing dawnward. The automatically determined cusp interval is $2244: 28-2251: 16 \mathrm{UT}$.

a few hours of local time in the direction controlled by the signs of IMF $B_{y}$ and $B_{z}$. This dispersion effect makes it difficult to study the location of the foot of the injection point. Therefore, it would be interesting if we have a more accurate estimation of the foot point of the reconnection line. For this purpose, we determined the location of the ion en- try point introduced earlier in this section for Cases 1 to 4 . It was located at $(-72.4,12.3)$ in Case 1 , at $(-82.3,12.2)$ in Case 2 , at $(78.4,10.4)$ in Case 3 , and at $(76.4,13.9)$ in Case 4 , as expressed in the latitude-local time coordinates. Comparing cases 1 and 2, we can see a clear dependence of the latitude of the ion entry point on IMF $B_{z}$. On the other 
PASS 91013107

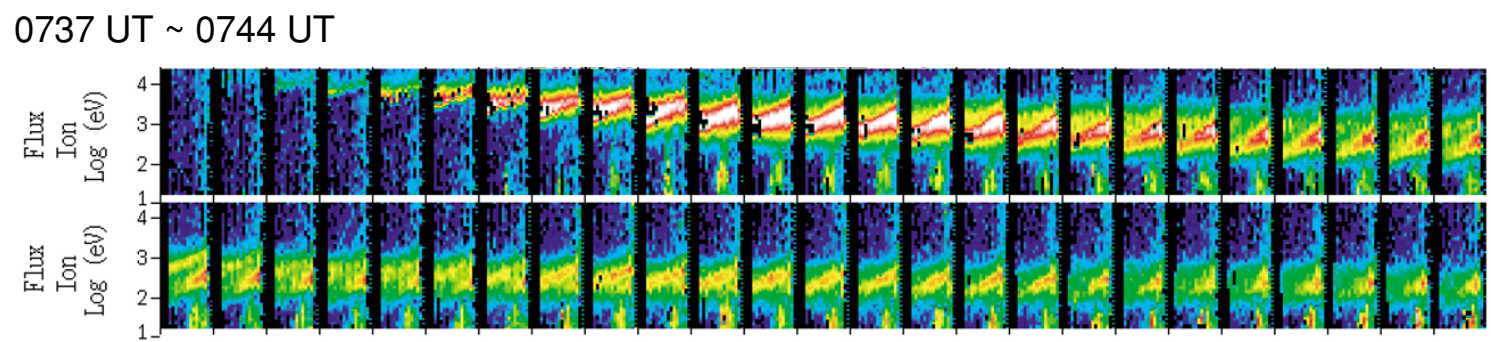

Fig. 6. The 8-sec ion spectrograms for Case 1 (Fig. 2) taken at $0737-0744$ UT. Horizontal axis represents pitch-angle $\left(180^{\circ}-0^{\circ}\right)$ of ions in each box. (Ions going upward correspond to $180^{\circ}$ and ions going upward correspond to $0^{\circ}$ because of the southern hemisphere).

Table 1. Automatically determined temporal and spatial (latitude, magnetic local time) ranges of the ion precipitation event, and the determined position of "ion entry point" for Cases $1-4$.

\begin{tabular}{cc|c|ccc|ccc|cc}
\hline & Date & IMF & \multicolumn{3}{c|}{ Event start } & \multicolumn{3}{c|}{ Event end } & \multicolumn{2}{c}{ "Ion entry point” } \\
& & & UT & LAT & MLT & UT & LAT & MLT & LAT & MLT \\
\hline 1 & 910131 & $B_{z}-$ & $0737: 56$ & -75.89 & 12.39 & $0744: 44$ & -72.35 & 12.25 & -72.43 & 12.25 \\
2 & 891005 & $B_{z}+$ & $0825: 40$ & -83.25 & 12.24 & $0830: 44$ & -79.32 & 11.98 & -83.25 & 12.24 \\
3 & 910725 & $B_{y}-$ & $2251: 56$ & 80.23 & 12.22 & $2258: 20$ & 78.36 & 10.42 & 78.36 & 10.42 \\
4 & 910806 & $B_{y}+$ & $2244: 28$ & 78.21 & 12.92 & $2251: 16$ & 76.27 & 13.95 & 76.36 & 13.91 \\
\hline
\end{tabular}

LAT vs IMF By

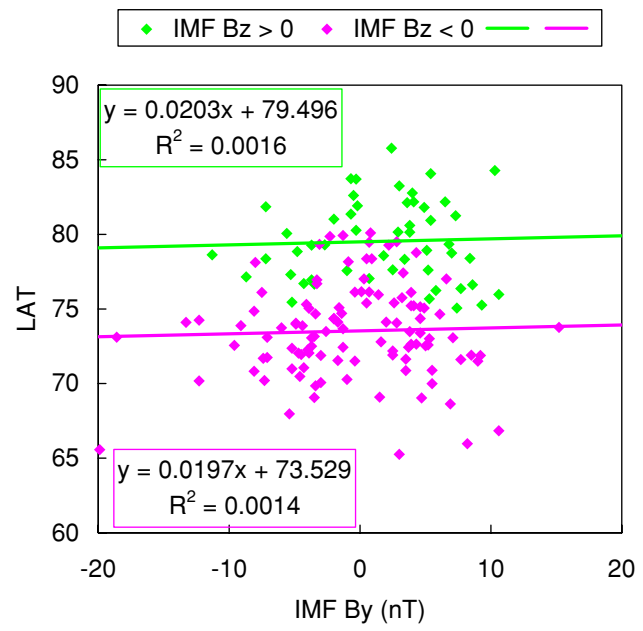

LAT vs IMF Bz

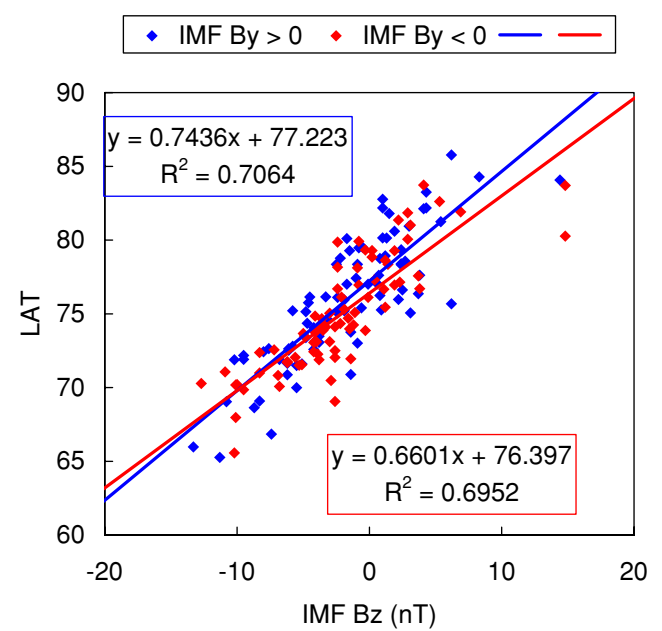

Fig. 7. The IMF dependence of the latitude (LAT) of the ion entry point. The left panel shows the latitude versus IMF $B_{y}$ plot. Green points are for the cases of positive $B_{z}$ and pink points are for the cases of negative $B_{z}$. The right panel shows the latitude versus IMF $B_{z}$ plot. Blue points are for the cases of positive $B_{y}$ and red points are for the cases of negative $B_{y}$. The colored lines show the regression lines for each IMF polarity.

hand, comparing cases 3 and 4, we see a clear dependence of the local time of the ion entry point on IMF $B_{y}$. In the following sections we will investigate the dependence of the location of the ion entry point on the IMF statistically using a large volume of data set obtained by Akebono.

\subsection{Cusp event selection}

The statistics of cusp-ion precipitation derive from more than 3,000 orbits of Akebono observations from 19891993, for which hourly IMF data were available. We believe that hourly values are appropriate for our correlational study because IMF effects on the magnetosphere require a finite time (of the order of tens of minutes) to establish itself in the form of convection. We excluded from our statistics the cases where either $B_{z}$ or $B_{y}$ changed sign from the previous hour in order to make our statistics more reliable.

Cusp precipitation intervals are automatically selected by a computer program according to the following criteria: (1) The average energy of precipitating ions does not exceed 3 $\mathrm{keV}$, (2) The average energy of precipitating electrons does not exceed $300 \mathrm{eV},(3)$ The number flux of precipitating ions exceeds $1 \times 10^{7} / \mathrm{cm}^{2} / \mathrm{sr} / \mathrm{sec}$, (4) The number flux of precipitating electrons exceeds $3 \times 10^{7} / \mathrm{cm}^{2} / \mathrm{sr} / \mathrm{sec}$. The first two criteria are for selecting precipitation events for which both electron and ion energies do not exceed the typical ranges of 

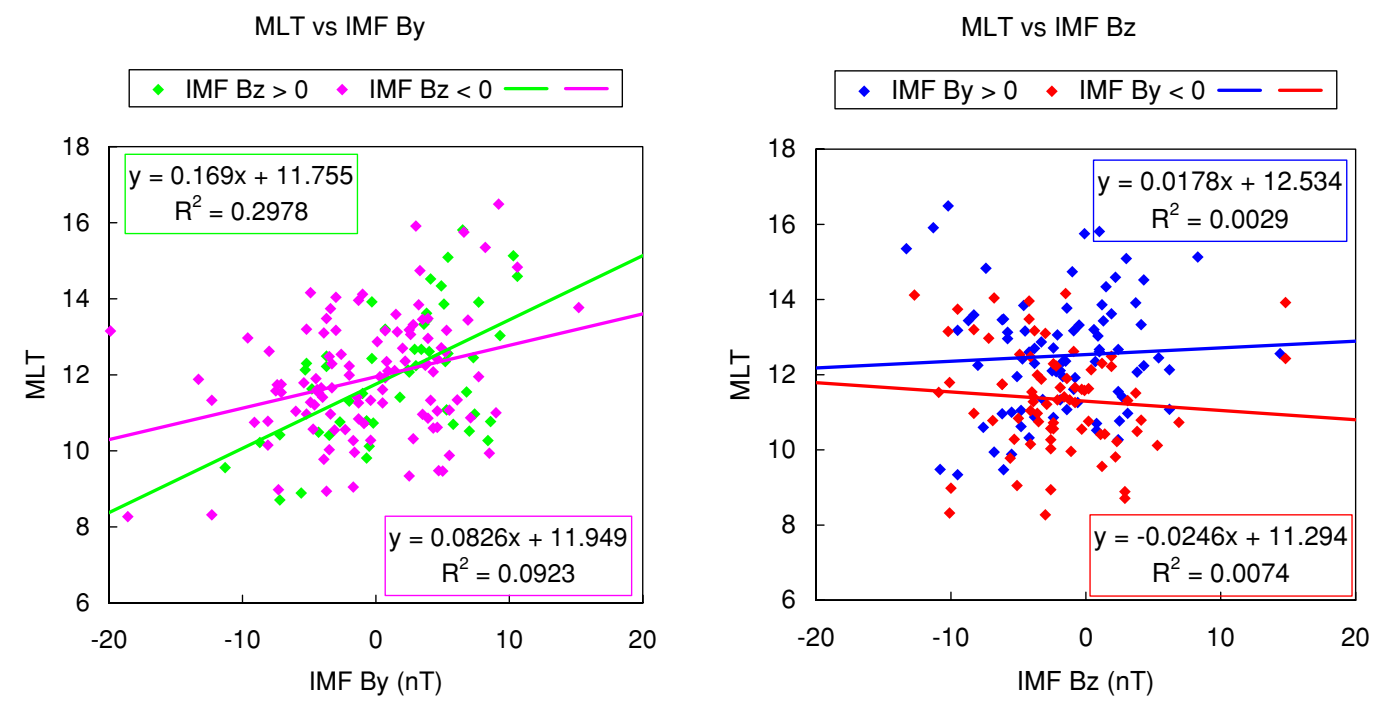

Fig. 8. Dependence of the magnetic local time (MLT) of the ion entry point on IMF, given in the same format as Fig. 7.
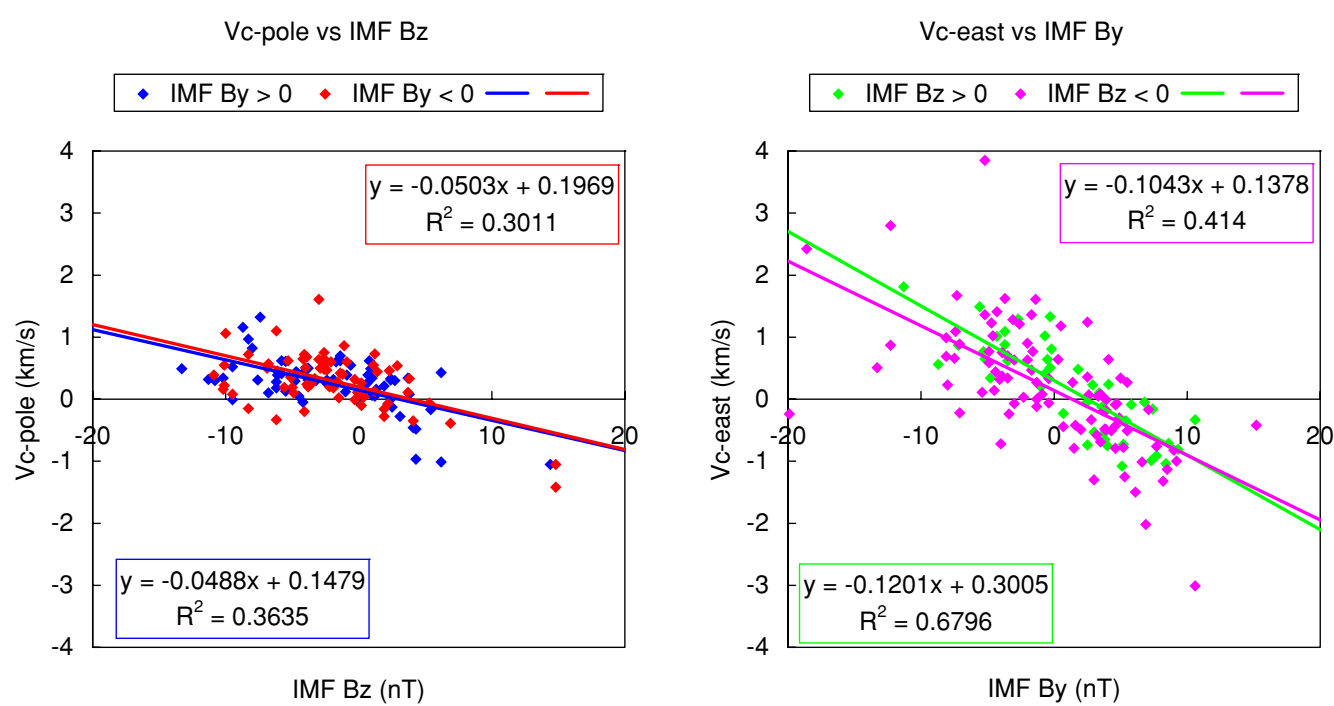

Fig. 9. The IMF dependence of the convection flow vectors estimated from the observed electric field: the poleward component of the convection velocity $(V p)$ is plotted against IMF $B_{z}$ (left) and the eastward components $(V e)$ is plotted against IMF $B_{y}$ (right). The colors have the same meanings as in Figs. 7 and 8.

the solar wind. These conditions are useful for distinguishing cusp from precipitation of magnetospheric origin. The latter two conditions are to avoid weak flux events for which the number of particle counts is close to the background noise level of the detector. Similar conditions have been imposed by Newell and Meng (1987) for identifying the lowaltitude cusp and its neighboring precipitation with DMSP data. For the present study, ion precipitation event is included in the analysis only when the above conditions were continuously satisfied during a period longer than $32 \mathrm{sec}$.

After a cusp precipitation event was automatically selected with a computer program, we required two additional criteria to be satisfied in order for the event to be included in the analysis. (5) The precipitation shows a clear ion dispersion signature spanning at least one order of magnitude in energy. (6) The pitch angle-energy dispersion signature is recognized in 8-sec resolution ion data. Condition (5) is employed to make the determination of the "ion entry point" in the energy dispersion curve easier and more reliable. In order to see the meaning of the condition (6), we show in Fig. 6 examples of 8-sec pitch angle-energy ion spectrograms for Case 1 (for the time interval of 0737-0744 UT). In each panel, the vertical axis represents ion energy and the horizontal axis represents ion pitch-angle $\left(180^{\circ}\right.$ for downward directed ions and $0^{\circ}$ for upward directed ions). Panels are displayed in the order of increasing time. The presence of pitch angle-dependent energy dispersion evidences that the ions have precipitated through magnetic flux tubes linked to the polar cusp; Burch et al. (1982) explained the pitch-angle energy dispersion in terms of the time-of-flight effect along the field lines assuming that the cusp ion source is narrow. Menietti and Burch (1988) calculated the fieldaligned distance of the source to be about $10 \mathrm{Re}$ from the observed point at an altitude of about $20,000 \mathrm{~km}$.

With the above six criteria, we identified 159 cusp precipitation events (73 in the southern hemisphere and 86 in 


\section{NORTH: total 79}
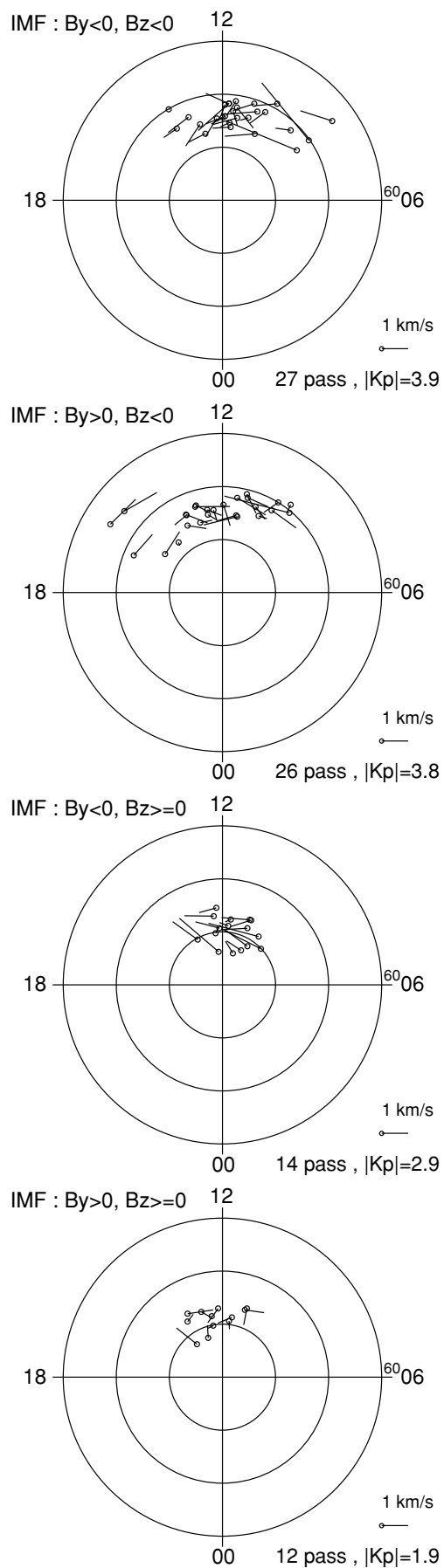

\section{SOUTH: total 58}
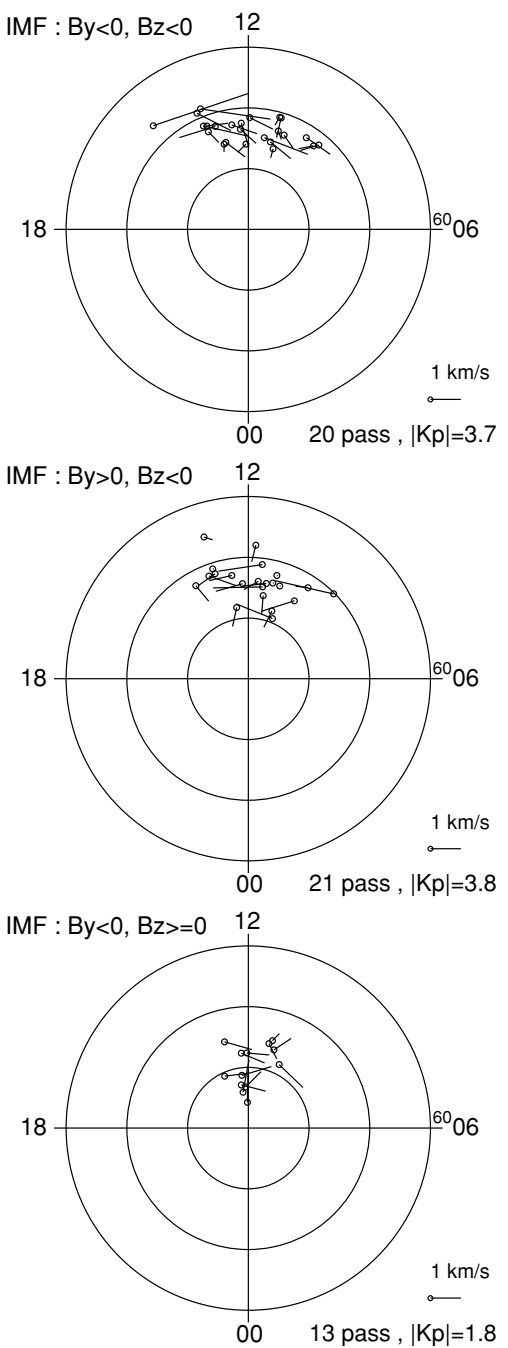

IMF : $\mathrm{By}>0, \mathrm{Bz}>=0 \quad 12$

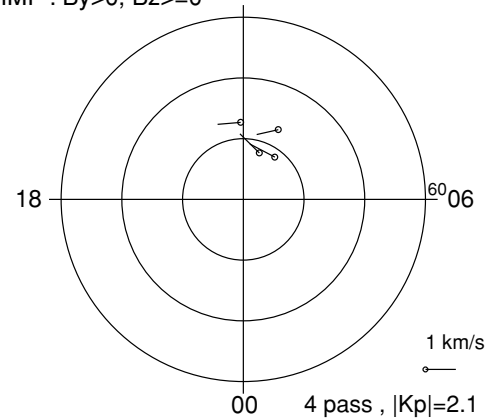

Fig. 10. Convection velocity vectors are plotted in such a way that they start from the observed positions of entry points (circles). Data for the northern hemisphere are shown on the left and data for the southern hemisphere on the right.

the northern hemisphere), and determined the location of the ion entry point for each event. Note that electron data were not used to identify the entry point because the energy range of cusp electron precipitation (about $100 \mathrm{eV}$ on average) is close to that of photoelectrons $(<70 \mathrm{eV})$. The time intervals and the spatial ranges of ion precipitation events as determined by the above criteria for Cases $1-4$ are listed in Table 1.

\subsection{Statistics}

We investigated the relationship between IMF conditions (in particular, $Y$ and $Z$ components) and the location of ion entry point for 159 cusp precipitation events.

Let us start with the latitude (LAT) and magnetic local time (MLT) of the entry point plotted in Figs. 7 and 8, respectively. The left panel of each figure shows the latitudes plotted against IMF $B_{y}$; the green points are for positive $B_{z}$, and the pink ones are for negative $B_{z}$. The right panels show the latitudes plotted against IMF $B_{z}$; the blue points are for 
positive $B_{y}$, and red ones are for negative $B_{y}$. The colored lines represent the linear regression lines for different IMF conditions.

The comparison of Cases 1 to 4 we made earlier indicated that the entry point is displaced in the longitudinal direction by IMF $B_{y}$ and in the latitudinal direction by IMF $B_{z}$. Since the longitudinal effect of IMF $B_{y}$ in the northern hemisphere is found to be opposite to that in the southern hemisphere, the southern and northern hemisphere events are plotted with the sign of IMF $B_{y}$ reversed and the mirror image of the satellite path is adopted with respect to the noon-midnight plane for the southern hemisphere events.

Figure 7 clearly shows that there is a clear linear relationship between the latitude of the ion entry point and IMF $B_{z}$ (see the right panel) but no relationship for IMF $B_{y}$ (see the left panel). As a mater of fact, the regression lines shown in the right panel are almost the same for positive and negative $B_{y}$. We conclude that IMF $B_{y}$ component does not influence the latitudinal position of the ion entry point.

On the other hand, Fig. 8 shows that the MLT (magnetic local time) of the ion entry point is related with IMF $B_{y}$; the entry point tends to occur at a later local time for more positive IMF $B_{y}$. Both two panels show that the MLT of the ion entry point has a weak $B_{z}$ dependence in such a way that the dependence on $B_{y}$ is larger for northward IMF than for southward IMF. This effect is addressed in the discussion section.

Note that the number of events for positive IMF $B_{z}$ is less than for negative IMF $B_{z}$. We suggest that this is due to one of our criteria used to select cusp ion precipitation, i.e. the condition that the energy dispersion should cover at least one order of magnitude in energy. We found that a significant portion of the cusp ion precipitation events for northward IMF does not show a clear energy dispersion and hence does not satisfy this condition.

\subsection{Convection with cusp precipitation}

Each of Case 1-4 shows clear IMF $B_{y}$ and $B_{z}$ effects on convection flows accompanied with cusp precipitation. The direction of convection flows projected onto the $120 \mathrm{~km}$ altitude level obtained from the electric field data is consistent with that predicted from the spatial energy dispersion of cusp ion precipitation. Figures 9 and 10 show the convection flows averaged over each event obtained from the 8 -sec electric field data. In Fig. 9, the poleward components $\left(V_{p}\right)$ of the convection velocity is plotted against IMF $B_{z}$ (left) and the eastward components $\left(V_{e}\right)$ of the convection velocity is plotted against IMF $B_{y}$ (right). The meaning of colors is the same as Figs. 7 and 8 . Figure 10 shows the same data in the form of the two-dimensional polar coordinate maps; northern hemisphere is shown to the left and the southern hemisphere is shown to the right. The small circles indicate the locations of the ion entry points and the attached vectors represent the convection velocity vectors for the corresponding events. We note that the electric field data were not available for some of the selected events so that the number of events (137) shown in Figs. 9 and 10 are less than that shown in Figs. 7 and 8. It is found from these plots that (1) the convection velocity tends to have a large azimuthal component, (2) the azimuthal component is almost linearly related to IMF $B_{y}$, (3) the cases of equatorward convec-
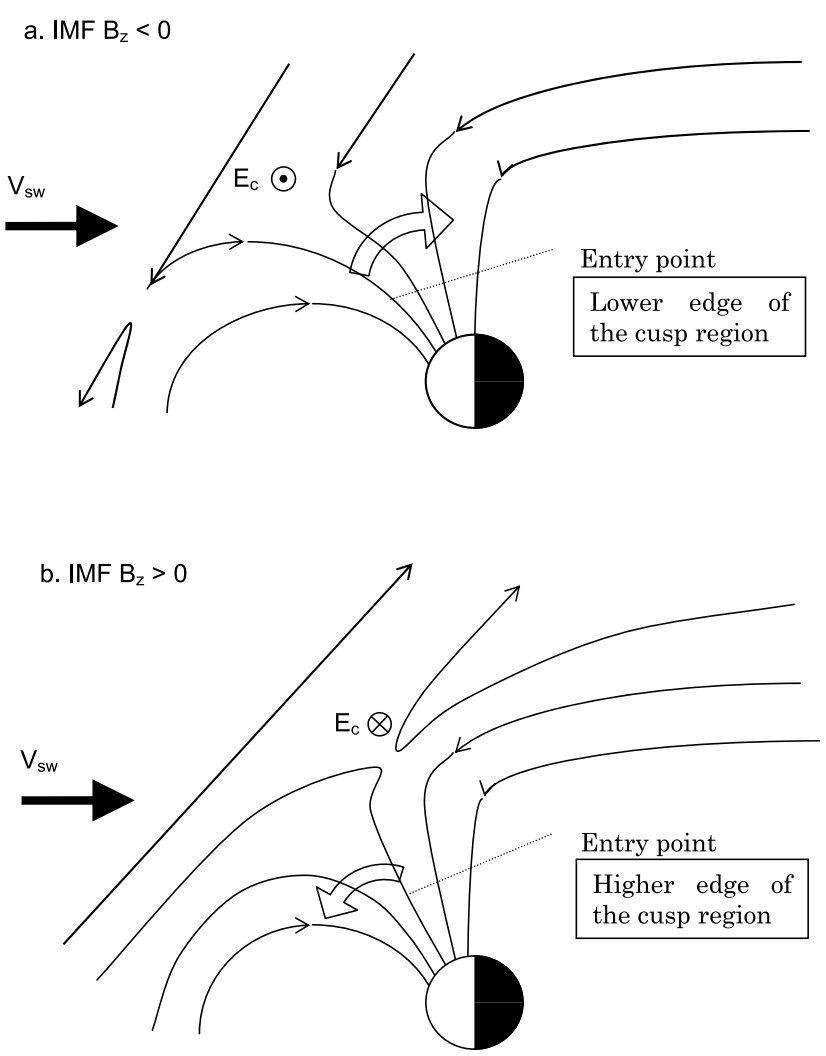

Fig. 11. Schematic diagram showing the difference of magnetic field configuration around the polar cusp between the northward and southward IMF cases. The ion entry point is regarded as the poleward (equatorward) edge of the polar cusp during northward (southward) IMF.

tion are few and appear only for strong northward IMF, and (4) poleward convection is observed for southward IMF and even for northward IMF in some cases.

\section{Discussion}

Since the ion entry point is probably associated with the magnetic foot point of the dayside reconnection line, we examine the location of the ion entry point and its dependence on IMF in order to obtain information about the dayside reconnection sites.

\subsection{Latitudinal displacement}

The ion entry point is regarded as the point nearest to the foot point of the separatrix of reconnected field lines, so that its latitudinal displacement is attributed to a property of the high-latitude reconnection when IMF $B_{z}>0$ and to a property of the low-latitude reconnection when $B_{z}<0$. In other words, the ion entry point for IMF $B_{z}>0$ is expected to represent the poleward edge of the polar cusp and that for IMF $B_{z}<0$ is expected to represent its equatorward edge. This suggestion has been made by Woch and Lundin (1992) based on Viking observations at altitudes $>12,000 \mathrm{~km}$, and by Basinska et al. (1992) based on DE2 observations at altitudes of $500 \mathrm{~km}$. They showed that the energy dispersion is due either to poleward or to equatorward convection. Onsager et al. (2001) have also reported an example of high-latitude reconnection observed by the Polar spacecraft at the altitude of about $6 \mathrm{Re}$. This suggestion is illustrated in Fig. 11 in which the magnetic field lines around the polar cusp are depicted for northward and southward 

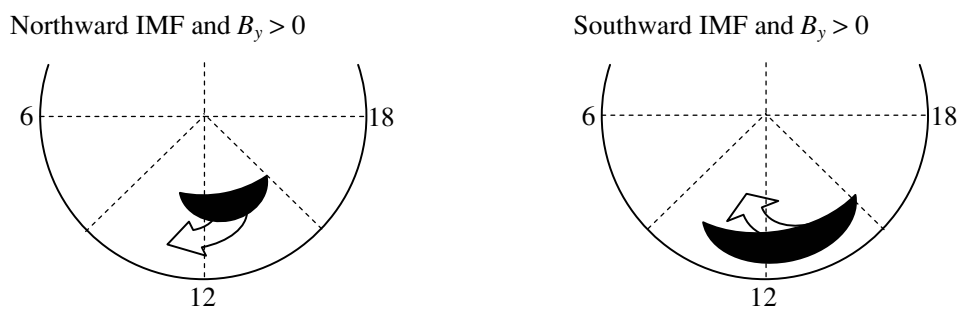

Fig. 12. Schematic diagrams showing the region of the ion entry point (solid areas) and the assumed convection flow direction (white arrows) for dominantly positive (negative) IMF $B_{y}$ in the northern (southern) hemisphere.

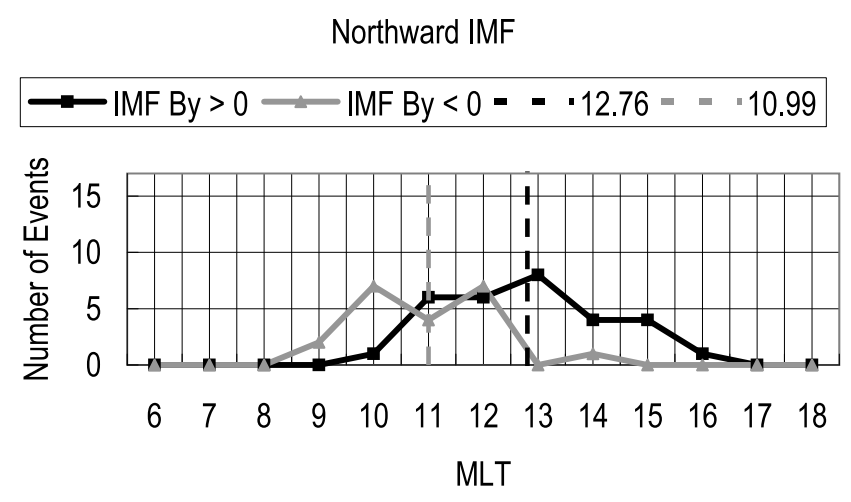

Southward IMF

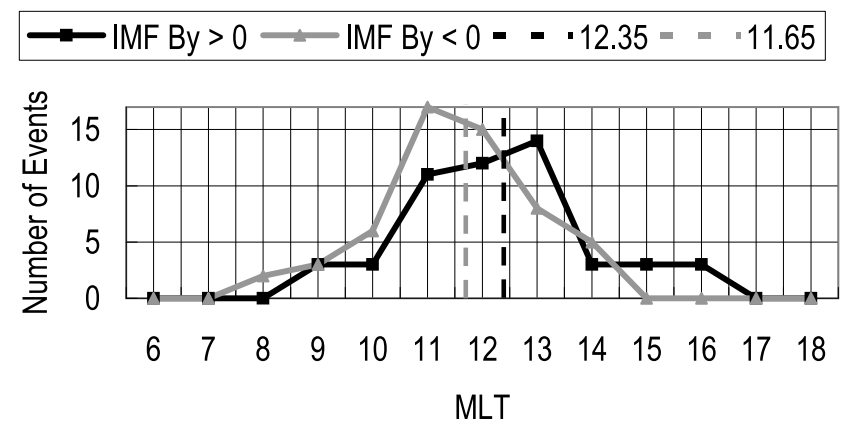

Fig. 13. The number of ion entry points as a function of MLT, the upper panel for northward IMF and the lower panel for southward IMF. For each panel, black lines show the cases with positive IMF $B_{y}$ and grey lines with negative IMF $B_{y}$.

IMF conditions. The relationship between the latitudinal position of the ion entry point and convection direction is easily understood in this figure. For southward IMF, terrestrial field lines are peeled off from the low-latitude magnetopause by the reconnection process occurring on the closed filed lines, so that the ion entry point moves toward lower latitudes, while the convection is directed poleward. On the other hand, for northward IMF, high-latitude reconnection occurs at the position tailward of the cusp so that the highlatitude field lines are peeled off and the ion entry point moves poleward while the convection is directed equatorward. The white arrows in Fig. 11 indicate the direction of convection.

Results shown in the right panel of Fig. 7 suggest that the dependence of the the ion entry point on $B_{z}$ can be expressed by a single linear regression line irrespective of the $B_{y}$ and $B_{z}$ signs. The best-fit regression formula for the distribution of all the 159 points is

$$
L A T[\mathrm{deg}]=0.71 B_{z}[\mathrm{nT}]+76.8 .
$$

The correlation coefficient is 0.83 . Note that our analysis is an average over different geomagnetic dipole tilt angles, and still a high degree of correlation has been obtained. The linear dependence suggests that the reconnection rate for the southward and northward IMF is not much different from each other. The continuity of the distribution of data points between the northward and southward $B_{z}$ ranges (see the right panel of Fig. 7) suggests that there is no wide gap separating the poleward and equatorward edges of the cusp. This would imply that the latitudinal width of the cusp is narrow, but it would be difficult to quantify the result at this moment.

In Paper 1 we already reported the presence of a good correlation between the latitude of ion entry point and IMF $B_{z}$. We found that the solar wind velocity weakly but clearly affects the latitudinal displacement of the ion entry point. It should be noted that the $V_{s w}$ effect is not in the form of the solar wind dynamic pressure but in the form of the solar wind electric field $\left(\boldsymbol{E}=-\boldsymbol{V}_{\boldsymbol{s w}} \times \boldsymbol{B}_{\mathrm{IMF}}\right)$ which acts to peel-off the terrestrial field lines. The dynamic pressure of the solar wind, $\rho V_{s w}^{2}$, directly controls the size of the magnetosphere but not the cusp latitude (see Sibeck et al., 1991, and Shue et al., 1997, for statistical analyses). Paper 1 also indicated that latitudinal saturation seems to occur at about $65^{\circ}$ and $85^{\circ}$ for electric fields exceeding $\pm 5 \mathrm{mV} / \mathrm{m}$.

Newell et al. (1989) reported the IMF effect on the position of the dayside cusp precipitation on the basis of data taken by DMSP satellites (at an altitude of $840 \mathrm{~km}$ ). However, in Newell's analysis, the energy dispersed nature of ion precipitation is not explicitly taken into account in the analysis of cusp location. They used for their analysis the position of the "cusp proper" where the number flux of ion precipitation was highest. They deduced different regression lines for different polarities of IMF $B_{z}$. On the other hand, our result suggests that the dependence can be expressed by a single regression line for entire range of IMF $B_{z}$. It is interesting that our regression line for the entire range of IMF $B_{z}$ is very close to Newell's regression line for southward IMF, i.e., LAT $[\mathrm{deg}]=0.76 B_{z}[\mathrm{nT}]+77.0$, but we regard the close similarity to be coincidental. It is also interesting to note that the correlation coefficient obtained from our analysis is significantly higher than that of Newell ( 0.70 for the southward IMF) in spite of the fact that our result includes both southward and northward $B_{z}$ cases. In our analysis, we narrowed the ambiguity of the location 

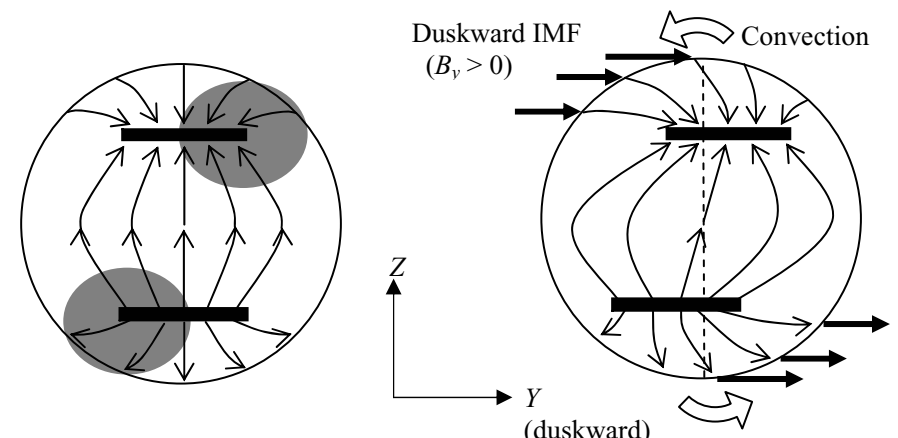

Fig. 14. Magnetic field line configuration on the dayside magnetopause as viewed from the sun. Undistorted magnetic field lines for the case of no IMF is shown in the left panel and a field line configuration distorted by the presence of duskward IMF (positive $B_{y}$ ) is shown in the right panel. The solid areas indicate the polar cusp, whose magnetic foot print would correspond to the ion entry points shown in Fig. 12.

of the cusp precipitation by introducing the concept of the ion entry point defined as the high-energy edge of the cusp ion precipitation. This definition is based on a theoretical consideration that it would represent the point closest to the foot point of the reconnection line along the satellite path. A high correlation coefficient obtained from our analysis may suggest that the latitudinal displacement of the ion entry point really reflects the latitudinal change of the starting point of reconnection and that our method worked well to remove the ambiguity due to the energy dispersion effects. Frey et al. $(2002,2003)$ referred to the Newell's result in the study of cusp proton aurora for southward IMF and not for northward IMF. Our result suggests that a single relationship between the cusp position and IMF $B_{z}$ applies for both northward and southward polarities.

\subsection{Longitudinal displacement}

Dayside convection is directed toward the pre-noon (post-noon) sector when IMF $B_{y}$ is positive (negative) in the northern hemisphere (Heelis, 1984; Gosling et al., 1990; etc.). Our results shown in Figs. 9 and 10 agree with these past studies. On the other hand, we found that the ion entry point defined in our paper shifts in the direction opposite to the convection direction, as shown in Fig. 8.

Figure 12 schematically shows the probable regions of the entry point (shaded areas) and the direction of the convection flow (white arrows) for dominantly positive (negative) IMF $B_{y}$ in the northern (southern) hemisphere (after Basinska et al., 2002). The right and left diagrams represent the cases for the negative and positive IMF $B_{z}$, respectively. Note that for the other polarity of $B_{y}$, i.e. for the cases of negative (positive) IMF $B_{y}$ in the northern (southern) hemisphere, the whole diagram becomes a mirror image about the noon meridian of Fig. 12. Our result suggests that the $B_{y}$ dependence of the ion entry point is consistent with the picture presented in Fig. 12. Our result also suggests that the ion entry point may be distributed in a longitudinally wide area, as is consistent with Fig. 12, since there is a large degree of scatter in magnetic local time plotted as a function of IMF $B_{y}$ in Fig. 8.

We noted earlier that the magnitude of the IMF $B_{y}$ effect on the azimuthal position of the ion entry point is different between the southward and northward IMF cases (see Fig. 8). To clarify this difference, Fig. 13 shows the MLT distribution of ion entry points for different polarities of IMF $B_{y}$ for northward (upper panel) and southward (lower panel) IMF conditions. In each panel, black lines are for positive $B_{y}$ and grey lines are for negative $B_{y}$. Colored vertical dashed lines in each panel indicate the average MLT position of the entry point for each IMF polarities. We can clearly see that the magnitude of the local-time shift of ion entry point induced by IMF $B_{y}$ is larger for northward $B_{z}$ than for southward $B_{z}$. In order to discuss the reason for this tendency, magnetic field line configuration on the dayside magnetopause is schematically shown in Fig. 14, as viewed from the sun (cf., Crooker et al., 1987; Stasiewicz, 1991; Fuselier et al., 2003). Undistorted magnetic field lines for the case of no IMF is shown on the left and a field line configuration distorted by the presence of positive IMF $B_{y}$ is shown on the right. The shaded areas in the left panel indicate the positions whose magnetic foot print would correspond to the region of ion entry point shown in Fig. 12. (Note that Fig. 12 is for the northern hemisphere.)

The sense of the $B_{y}$-related shift of the polar cusp has been interpreted in the literature in the following way. Magnetospheric magnetic field lines on the magnetopause around the polar cusp have diverse directions, so that it is easy for them to satisfy the anti-parallel field condition, which has been proposed by some researchers to be a necessary condition for magnetic reconnection. When IMF $B_{y}$ is positive, the shadowed areas in the left diagram are a candidate for the site of anti-parallel reconnection. In the northern hemisphere, for example, the shadowed area is on the duskside of the polar cusp, and the magnetic field lines on the duskside are peeled-off, and therefore the ion entry point moves toward dusk. This movement of the ion entry point is opposite to the convection direction shown by white arrows in Fig. 12 (and also shown in the right panel of Fig. 14), and is consistent with our statistics.

In conclusion, our result on the IMF $B_{y}$ dependence of ion entry point is consistent with the concept of anti-parallel reconnection. However, we should point out an important possibility that our result is also consistent with the concept of component merging. Crooker et al. (1997) studied on high latitude merging sites during periods of large IMF $B_{y}$ and Crooker et al. (1998) indicated by numerical simulation that the polar cusp is displaced by IMF $B_{y}$. The right panel 
of Fig. 14 shows the distorted magnetospheric field lines on the magnetopause based on their simulation. The magnetospheric magnetic field lines are distorted because the IMF $B_{y}$ component penetrates into the magnetosphere as a result of reconnection. We should point out that the sufficient condition for this kind of field line distortion is that IMF $B_{y}$ penetrates into the magnetosphere, and the condition is not related to the question of whether the anti-parallel condition or component merging condition is satisfied at the reconnection point. Once the magnetospheric field lines are distorted in the way depicted in the right panel of Fig. 14, it would be a easy matter to see that the foot point of the dayside low latitude field lines, on which the component merging is assumed to occur, is also shifted toward dusk in the northern hemisphere and toward dawn in the southern hemisphere, in a way similar to the case of anti-parallel reconnection.

The above discussion implies that our results (as well as other similar results in literature) do not discriminate between anti-parallel and component merging. We actually conjecture that reconnection can occur both on the lowlatitude field lines and on the cusp field lines when IMF $B_{z}$ is close to zero. The probability of high-latitude reconnection would become higher for northward IMF and the probability of low-latitude reconnection would become higher for southward IMF. The stronger $B_{y}$ dependence of the MLT position of the ion entry point for northward IMF would then mean that the $B_{y}$ dependence of the position of the anti-parallel merging site around the cusp is stronger than the field line distortion effect associated with the lowlatitude reconnection. We should also consider the fact that the low latitude reconnection line for the case of southward IMF is expected to be extended over a wide range of MLT so that the local time position of the ion entry point may have a large scatter, as is consistent with the result shown in Fig. 8.

\section{Summary}

From the analysis of ion precipitation events observed by the Akebono satellite, we have statistically investigated latitudinal and longitudinal displacements of cusp precipitation controlled by IMF. We introduced the concept of "ion entry point" which is defined to be the point where the precipitation ion energy was the maximum along the satellite path. The ion entry point is interpreted to be the point nearest to the magnetic foot point of the dayside magnetic reconnection line along the satellite path.

Our analysis showed that the location of the ion entry point has a strong dependence on the sign and magnitude of IMF $B_{y}$ and $B_{z} . \quad B_{y}$ and $B_{z}$ have almost orthogonal effects on the location of the entry point, with the latitudinal displacement linearly related to $B_{z}$, and the longitudinal displacement linearly related to $B_{y}$. We have found that the $B_{z}$ dependence of the ion entry point can be represented by a single regression line for entire range of $B_{z}$ irrespective of its north/south polarity. We have also found that the $B_{y}$ dependence is larger for positive $B_{z}$ than for negative $B_{z}$. The distribution of ion entry points for positive IMF $B_{y}$ is almost a mirror image of that for negative IMF $B_{y}$ with respect to the noon-midnight meridian plane. We discussed the dependence of the ion entry point on the IMF from the viewpoint of where magnetopause reconnection (merging) occurs on the dayside magnetosphere and how the field lines are distorted by the reconnection process. We conclude that the observed dependence of the ion entry point on IMF $B_{y}$ is consistent with both the anti-parallel and component merging models of the dayside reconnection.

Acknowledgments. The solar wind and IMF data used in this analysis were provided by NSSDC.

\section{References}

Aparicio, B., B, Thelin, and R. Lundin, The polar cusp from a particle point of view: A statistical study based on Viking data, J. Geophys. Res., 96, 14023-14031, 1991

Asai, K., K. Maezawa, N. Kaya, and T. Mukai, Latitudinal displacement of cusp ion precipitation: Akebono observations, Adv. Space Res., 23, 1761-1764, 1999.

Basinska, E. M., W. J. Burke, N. C. Maynard, W. J. Hughes, J. D. Winningham, and W. B. Hanson, Small-scale electrodynamics of the cusp with northward Interplanetary Magnetic Field, J. Geophys. Res., 97, 63696379, 1992.

Burch, J. L., P. H. Reiff, R. A. Heelis, J. D. Winningham, W. B. Hanson, C. Gurgiolo, J. D. Menietti, R. A. Hoffman, and J. N. Barfield, Plasma injection and transport in the mid-altitude polar cusp, Geophys. Res. Lett., 9, 921-924, 1982.

Chandler, M. O., S. A. Fuselier, M. Lockwood, and T. E. Moore, Evidence of component merging equatorward of the cusp, J. Geophys. Res., 104, 22,623-22,634, 1999.

Cowley, S. W. H., The couses of convection in the Earth's magnetosphere: A review of developments during IMS, Rev. Geophys., 20, 531-565, 1982.

Cowley, S. W. H., J. P. Morelli, and M. Lockwood, Dependence of convective flows and particle precipitation in the high-latitude dayside ionosphere on the $\mathrm{X}$ and $\mathrm{Y}$ components of the interplanetary magnetic field, J. Geophys. Res., 96, 5557-5564, 1991.

Crooker, N. U., J. Berchem, and C. T. Russell, Cusp displacement at the magnetopause for large IMF Y component, J. Geophys. Res., 92, 13467-13471, 1987.

Crooker, N. U., J. G. Lyon, and J. A. Fedder, MHD model merging with IMF $B_{y}$ : Lobe cells, sunward polar cap convection, and overdraped lobes, J. Geophys. Res., 103, 9143-9152, 1998.

Farrugia, C. J., P. E. Sandholt, R. B. Torbert, and N. Østgaard, Temporal and spatial aspects of the cusp inferred from local and global groundand space-based observations in a case study, J. Geophys. Res., 109, A04209, 2004.

Frey, H. U., S. B. Mende, T. J. Immel, S. A. Fuselier, E. S. Claflin, J.-C. Gérard, and B. Hubert, Proton aurora in the cusp, J. Geophys. Res., 107, 1091, 2002.

Frey, H. U., S. B. Mende, S. A. Fuselier, T. J. Immel, and N. Østgaard, Proton aurora in the cusp during southward IMF, J. Geophys. Res., 108, 1277, 2003.

Fuselier, S. A., H. U. Frey, K. J. Trattner, S. B. Mende, and J. L. Burch, Cusp aurora dependence on interplanetary magnetic field $B_{z}, J$. Geophys. Res., 107, 1111, 2002.

Fuselier, S. A., S. B. Mende, T. E. Moore, H. U. Frey, S. M. Petrinec, E. S. Claflin, and M. R. Collier, Cusp dynamics and ionospheric outflow, Space Science Reviews, 109, 285-312, 2003.

Gosling, J. T., M. F. Thomsen, S. J. Bame, R. C. Elphic, and C. T. Russell, Plasma flow reversals at the dayside magnetopause and the origin of asymmetric polar cap convection, J. Geophys. Res., 95, 8073-8084, 1990.

Hakura, Y., Tables and maps of geomagnetic coordinates corrected by the higher order spherical harmonic terms, Rep. Ionos. Space Res. Japan, 19, 121-157, 1965.

Hayakawa, H., et al., Electric field measurement on the Akebono (EXOSD) satellite, J. Geomag. Geoelectr., 42, 371-384, 1990.

Heelis, R. A., The effects of interplanetary magnetic field orientation on dayside high-latitude ionospheric convection, J. Geophys. Res., 89 , 2873-2880, 1984.

Karlson, K. A., M. Oieroset, J. Moen, and P. E. Sandholt, A statistical study of flux transfer event signatures in the dayside aurora: The IMF $B_{y}$-related prenoon-postnoon asymmetry, J. Geophys. Res., 101, 59-68, 
1996.

Kremser, G. and R. Lundin, Average spatial distributions of energetic particles in the midaltitude cusp/cleft region observed by Viking, $J$. Geophys. Res., 95, 5753-5766, 1990.

Lockwood, M., Relationship of dayside precipitation to the open-closed separatrix and the pattern of convection flow, J. Geophys. Res., 102, 17475-17487, 1997.

Lockwood, M., S. W. Cowley, P. E. Sandholt, and U. P. Lovhaug, Causes of plasma flow bursts and dayside auroral transients: an evaluation of two models invoking reconnection pulses and chaned in the Y component of the magnetosheath field, J. Geophys. Res., 100, 7613-7626, 1995.

Lockwood, M., S. W. H. Cowley, and T. G. Onsager, Ion acceleration at both the interior and exterior Alfven waves associated with the magnetopause reconnection site: Signatures in cusp precipitation, J. Geophys. Res., 101, 21501-21513, 1996.

Maezawa, K., Magnetospheric convection induced by the positive and negative $\mathrm{Z}$ components of the interplanetary magnetic field: quantitative analysis using polar cap magnetic records, J. Geophys. Res., 81, 2289_ 2303, 1976.

Maynard, N. C., W. J. Burke, P. E. Sandholt, J. Moen, D. M. Ober, M. Lester, D. R. Weimer, and A. Egeland, Observations of simultaneous effects of merging in both hemispheres, J. Geophys. Res., 106, 2455124578, 2001

Menietti, J. D. and J. L. Burch, Spatial extent of the plasma injection region in the cusp-magnetosheath interface, J. Geophys. Res., 93, 105-113, 1988.

Mukai, T., N. Kaya, E. Sagawa, M. Hirahara, W. Miyake, T. Obara, H. Miyaoka, S. Machida, H. Yamagishi, M. Ejiri, H. Matsumoto, and T. Itoh, Low energy charged particle observation in the "auroral" magnetosphere: First results from Akebono (EXOS-D) satellite, J. Geomag. Geoelectr., 42, 479-496, 1990.

Newell, P. T. and C.-I. Meng, Cusp width and $B_{z}$ : Observations and conceptual model, J. Geophys. Res., 92, 13673-13678, 1987.

Newell, P. T. and C.-I. Meng, The cusp and the cleft/boundary layer: Lowaltitude identification and statistical local time variation, J. Geophys. Res., 93, 14549-14556, 1988.

Newell, P. T., C.-I. Meng, D. G. Sibeck, and R. Lepping, Some low-altitude cusp dependencies on the interplanetary magnetic field, J. Geophys. Res., 94, 8921-8927, 1989.

Newell, P. T., W. J. Burke, C.-I. Meng, E. R. Sanches, and M. E. Greenspan, Identification and observations of the plasma mantle at low altitude, J. Geophys. Res., 96, 35-45, 1991a.

Newell, P. T., W. J. Burke, C.-I. Meng, E. R. Sanches, M. E. Greenspan, and C. R. Clauer, The low-latitude boundary layer and the boundary plasma sheet at low altitude: Prenoon precipitation regions and convection reversal boundaries, J. Geophys. Res., 96, 21013-21023, 1991 b.
Onsager, T. G., C. A. Kletzing, J. B. Austin, and H. MacKiernan, Model of magnetosheath plasma in the magnetosphere: Cusp and mantle particles at low-altitudes, Geophys. Res. Let., 20, 479-482, 1993.

Onsager, T. G., S.-W. Chang, J. D. Perez, J. B. Austin, and L. X. Janoo, Low-altitude observations and modeling of quasi-steady magnetopause reconnection, J. Geophys. Res., 100, 11831-11843, 1995.

Onsager, T. G., J. D. Scudder, M. Lockwood, and C. T. Russell, Reconnection at the high-latitude magnetopause during northward interplanetary magnetic field conditions, J. Geophys. Res., 106, 25467-25488, 2001.

Reiff, P. H., T. W. Hill, and J. L. Burch, Solar wind plasma injection at the dayside magnetospheric cusp, J. Geophys. Res., 82, 479-491, 1977.

Ridley, A. J., G. Lu, C. R. Clauer, and V. O. Papitashvili, A statistical study of the ionospheric convection response to changing interplanetary magnetic field conditions using the assimilative mapping of ionospheric electrodynamics technique, J. Geophys. Res., 103, 4023-4040, 1998.

Rosenbauer, H., H. Grunwaldt, M. D. Montgomery, G. Paschmann, and N. Sckopke, Heos 2 plasma observations in the distant polar magnetosphere: the plasma mantle, J. Geophys. Res., 80, 2723-2737, 1975.

Sandholt, P. E. and C. J. Farrugia, Does the aurora provide evidence for the occurrence of antiparallel magnetopause reconnection?, J. Geophys. Res., 108, 1466, 2003.

Shue, J.-H., J. K. Chao, H. C. Fu, C. T. Russell, P. Song, K. K. Khurana, and H. J. Singer, A new functional form to study the solar wind control of the magnetopause size and shape, J. Geophys. Res., 102, 9497-9511, 1997.

Sibeck, D. G., R. E. Lopez, and E. C. Roelof, Solar wind control of the magnetopause shape, location, and motion, J. Geophys. Res., 96, 54895495, 1991.

Stasiewicz, K., A global model of gyroviscous field line merging at the magnetopause, J. Geophys. Res., 96, 77-86, 1991.

Stubbs, T. J., P. J. Cargill, M. Lockwood, M. Grande, B. J. Kellett, and C. H. Perry, Extended cusp-like regions and their dependence on the Polar orbit, seasonal variations, and interplanetary conditions, J. Geophys. Res., 109, A09210, 2004.

Twitty, C., T. D. Phan, G. Paschmann, B. Lavraud, H. Rème, and M. Dunlop, Cluster survey of cusp reconnection and its IMF dependence, Geophys. Res. Lett., 31, L19808, 2004.

Woch, J. and R. Lundin, Magnetosheath plasma precipitation in the polar cusp and its control by the interplanetary magnetic field, J. Geophys. Res., 97, 1421-1430, 1992.

K. T. Asai (e-mail: asaikt@nict.go.jp), K. Maezawa, T. Mukai, and H. Hayakawa 